\title{
Opportunistic Screening for Detection and Socio-epidemiological Risk Assessment of Oral Cancer Patients in Rural Jodhpur, India
}

\begin{abstract}
Background: Globally, India has a high burden (20\%) of oral cancer with $1 \%$ prevalence of premalignant lesions. Most cases are attributed to modifiable risk factors such as substance abuse (tobacco and alcohol), dietary deficiencies, and environmental exposures (solar radiation and air pollution) aggravated by delayed detection and care especially in rural areas. Objective: The objective of the study was to study the risk factors of oral cancer pathogenesis among the rural residents of Jodhpur, India, through opportunistic oral screening approach at primary care facilities. Methodology: An unmatched case-control study was done at two randomly chosen rural health centres in Jodhpur, India. A total of 84 cases and 168 controls were included during 6 months study period (2016). Randomly selected outpatient department attendees were interviewed and screened for oral cancer and premalignant lesions. A structured questionnaire interview along with comprehensive oral, head and neck examination was conducted. Data were analyzed using multivariate logistic regression, and confidentiality of data was maintained. Results: The majority of the study participants were rural residents $(82.9 \%)$ with poor socioeconomic status. Opportunistic oral screening revealed a variety of cancerous and precancerous lesions. Most common case pathologies were submucosal fibrosis (40.5\%), inadequate mouth opening (35.7\%), cheek bites (28.6\%), leukoplakia (23.8\%) etc. Multivariate analysis suggested that tobacco intake (adjusted odds ratio $=13.6, P \leq 0.01$ ) dietary deficiency $(7.4,<0.01)$, oral sepsis $(7.0,<0.01)$, oral lesions $(6.8,<0.01)$, and sun radiation exposure $(9.5,<0.01)$ were significantly associated with oral cancer pathology. Conclusion: The study provides strong evidence that tobacco, dietary deficiency, oral sepsis and lesions, and sun radiation exposure are independent risk factors for oral cancer. It also reiterates the importance and application of opportunistic oral cancer screening at primary care level.
\end{abstract}

Keywords: Case control, India, NPCDCS, Opportunistic screening, Oral cancer, Oral pathology, Tobacco

\section{Introduction}

Modern epidemic of noncommunicable diseases continues to plague India's health care due to its enormous economic and social burden. Among the afflicted, rural residents remain severely disadvantaged due to poor accessibility and affordability of health care. India has world's highest burden $(20 \%)$ of oral cancers with $1 \%$ of the Indian population having oral premalignant lesions. Each year approximately 1 million people in India are diagnosed with oral cancers and half of them die a painful death within 12 months of diagnosis due to late presentation and inadequate care. ${ }^{[1]}$ Most cases of oral cancer are attributable to modifiable risk factors such as tobacco and alcohol consumption and combined risk multiplication. The odds of developing

This is an open access journal, and articles are distributed under the terms of the Creative Commons Attribution-NonCommercial-ShareAlike 4.0 License, which allows others to remix, tweak, and build upon the work non-commercially, as long as appropriate credit is given and the new creations are licensed under the identical terms.

For reprints contact: reprints@medknow.com oral cancer increases with frequency and duration of such exposures. ${ }^{[2]}$ Other known risk factors include: oral lesions, chronic oral sepsis, gender, age, photo radiation, poor nutrition, immunodeficiency states, familial or genetic predisposition, and air pollution. ${ }^{[3]}$

The Indian tobacco industry leads in the production of pan masala and gutkha (sweetened areca nut and chewing tobacco) and bidis (a form of handmade cigarette), especially to rural markets in the subcontinent. Habitual chewing of pan masala/gutkha is significantly associated with oral cancer pathogenesis including premalignant lesions. ${ }^{[4]}$ Approximately $90 \%$ oral cancers in southeast Asia are linked to tobacco chewing and smoking. According to the latest National Family Health Survey (NFHS 4, 2015-2016), prevalence of tobacco consumption among men and women in India were $44.5 \%$ and $6.8 \%$,

How to cite this article: Meena JK, Verma A,
Upadhyay SK. Opportunistic screening for detection
and socio-epidemiological risk assessment of oral
cancer patients in rural Jodhpur, India. Indian J Med
Paediatr Oncol 2018;39:452-5.

\section{Jitendra Kumar Meena, Anjana Verma, Sandeep Kumar Upadhyay ${ }^{1}$}

Department of Community Medicine, Geetanjali Medical College and Hospital, Udaipur, ${ }^{1}$ Department of Community and Family Medicine, All India Institute of Medical Sciences, Jodhpur, Rajasthan, India

Address for correspondence: Dr. Jitendra Kumar Meena, Department of Community Medicine, Geetanjali Medical College and Hospital, Udaipur - 313 002, Rajasthan, India.

E-mail: drmeenajk@gmail.com

Access this article online

Website: www.ijmpo.org

DOI: 10.4103/ijmpo.ijmpo_90_17

Quick Response Code:

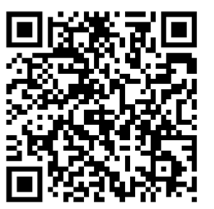


respectively. Similarly, alcohol consumption rates were $29.2 \%$ and $1.2 \%$ among men and women. ${ }^{[5]}$

Most oral cancers are often detected in advanced stages ; hence, treatment does not offer significant improvement in survival rates. It has been reported that late detection decreases the 5-year survival rate to $<50 \%$ for tongue and floor of mouth cancers. ${ }^{[6]}$ The advanced disease requires aggressive therapy with various treatment combinations that usually results in adverse effects, increased cost of care and reduced quality of life. The problem is not simply the stagnation or rise in number of new cancer cases but prevailing risk behaviors in public largely attributed to poor awareness. According to latest National Family Health Survey (NFHS-4) data only $12.4 \%$ Indian women have ever undergone an oral examination in their lifetime. ${ }^{[5]}$ If the current trends are not checked, an estimated 500 million people alive today will be killed by reasons associated with tobacco intake primarily oral cancer pathology. ${ }^{[7]}$

India has committed to screen the major three cancers (breast, cervical, and oral) through National Cancer Control Programme (NCCP) now integrated into National Programme for Prevention and Control of Cancers, Diabetes, Cardiovascular diseases, and Stroke (NPCDCS). However, due to poor infrastructure and unavailability of specialists, program implementation remains dismal. ${ }^{[8]}$ Therefore, the development of practical and sustainable oral screening infrastructure in primary health care is highly pertinent in today's scenario. Adopting best practice in soft-tissue screening will not only lead to the detection of oral cancer pathogenesis at an earlier stage but also facilitate appropriate treatment at the earliest. Present study aims at filling the existing knowledge gap regarding oral cancer epidemiology in jodhpur region. This study envisages further investigating the association of various socio-epidemiological risk factors with oral cancers and premalignant lesions.

\section{Methodology}

It was an unmatched case-control study done at two randomly selected rural health centers (PHC Dhawa and CHC Dhundhara) in the Jodhpur district of India. The study was conducted at general outpatient departments (OPDs) of health centers for 6 months in year 2016. A study sample comprising 84 cases and 168 controls (odds ratio [OR]: 4, exposure control: 0.06) was considered based on expert opinion. The study was done on randomly chosen OPD attendants meeting the inclusion criteria and consenting participation.

Chosen attendees were subsequently interviewed and screened for oral cancer and premalignant lesions. A predesigned, pretested questionnaire was used, and a comprehensive oral, head and neck examination conducted as part of screening activity. The questionnaire was validated by both internal and external experts. The questionnaire comprised of sociodemographic data such as age, gender, literacy, occupation, address, medical history date of diagnosis, and site (if lesion present). In addition, the questionnaire included questions on oral hygiene practices and oral cancer pathology risk factors, comprising of questions on type, frequency, and duration of exposure. The investigating physician underwent specialized training in the detection of oral pathologies and standard operating procedures for opportunistic oral cancer screening. To ensure completeness, examination followed a logical sequence involving an extra and intraoral examination. The visual inspection was supplemented by palpating suspicious lesions and lymph nodes (submandibular and cervical). Confirmation of suspected cases was done on the basis of histopathological biopsy test and available medical records.

A case was defined as an adult OPD attendee (aged 18 years and above) with laboratory confirmed oral cancer pathology and investigated based on screening and medical records. A control was defined as an adult OPD attendee who did not have any present signs or history of any oral cancer pathology during screening. The eligible attendees who did not consent were not included in the study. People with any other malignancy and/or people suffering from any disease associated with the study exposures were excluded from the study.

All records entered were cross verified and randomly double checked for correctness. Data collection and compilation involved a strict 2-stage quality mechanism including inbuilt checks with $10 \%$ random data revision. Informed consents were taken from all study participants; data confidentiality was maintained at all stages. Ethical approval for the study was obtained from the Institutional Ethical Committee of AIIMS, Jodhpur. Data were analyzed using Statistical Package for the Social Sciences software (version 21, Armonk, NY: IBM Corp) considering a $P<0.05$ as significant. Univariate analysis was done followed by multivariate logistic regression for identifying the risk association, adjusted for the confounding variables.

\section{Results}

The majority of the participants were rural residents $(82.9 \%)$, and most being males $(69.7 \%)$. The mean age of the participants was 48.6 years, education (3.2 years) and monthly income being 1430 rupees.

Only modifiable risk factor data were considered for inclusion in the study results for a clearer understanding of their association with oral cancer pathogenesis and drafting recommendations. Most known risk factors had a higher prevalence in the case group; however, some risk factors also had relatively high occurrence in control group. Risk factors such as tobacco intake (88.1\%), dietary deficiency $(82.1 \%)$, poor oral hygiene $(35.7 \%)$, sun radiation exposure $(46.4 \%)$, pollution exposure $(22.6 \%)$, pastoral lesions $(20.2 \%)$, and sepsis (17.9\%) were 
relatively abundant in the case group. Conversely, high salt intake (45.8\%) and alcohol intake $(27.9 \%)$ were found more frequently in the control group [Table 1].

Subsequently, sorted analysis of the oral examination findings (cases) revealed a variety of cancerous and precancerous lesions. Most common pathologies detected were submucosal fibrosis (40.5\%), inadequate mouth opening (35.7\%), cheek bites (28.6\%), and leukoplakia (23.8\%). Other pathological findings were $\mathrm{red} /$ white patch $(19.0 \%)$, lichen planus (16.7\%), tooth mobility (14.3\%), ulcer $(9.5 \%)$, failure to heal $(9.5 \%)$, fixation $(4.8 \%)$, growth $(2.4 \%)$, and pain or paresthesias (2.4\%) [Table 2].

A multivariate logistic regression analysis was performed to ascertain the effects of risk factors under study to the likelihood of oral cancer pathogenesis. Forward selection of the variables was done for induction in the model, postunivariate assessment of their risk significance. Participants who consumed tobacco $(\mathrm{P} \leq 0.01)$ had 13.6 times risk of oral cancer pathology compared to those who did not. Other risk factors, namely, Dietary deficiency (adjusted OR $=7.4, P \leq 0.01$ ), oral sepsis (7.0, $<0.01)$, oral lesions $(6.8,<0.01)$, and sun radiation exposure $(9.5,<0.01)$ demonstrated significantly high

\begin{tabular}{|c|c|c|}
\hline \multicolumn{3}{|c|}{$\begin{array}{l}\text { Table 1: Risk factor distribution among the study } \\
\text { participants }\end{array}$} \\
\hline \multirow[t]{2}{*}{ Risk factor } & \multicolumn{2}{|c|}{ Prevalence, $n(\%)$} \\
\hline & Cases $(n=84)$ & Controls $(n=168)$ \\
\hline Tobacco intake & $74(88.1)$ & $59(35.1)$ \\
\hline Dietary deficiency & $69(82.1)$ & $64(38.1)$ \\
\hline High salt intake & $37(44.0)$ & $77(45.8)$ \\
\hline Poor oral hygiene & $30(35.7)$ & $46(27.3)$ \\
\hline Sun radiation exposure & $39(46.4)$ & $14(8.3)$ \\
\hline Alcohol intake & $19(22.6)$ & $47(27.9)$ \\
\hline Pollution exposure & $19(22.6)$ & $26(15.4)$ \\
\hline Oral lesions & $17(20.2)$ & $6(3.6)$ \\
\hline Oral sepsis & $15(17.9)$ & $5(3.0)$ \\
\hline
\end{tabular}

*Items are not mutually exclusive

\begin{tabular}{lc}
\hline \multicolumn{1}{c}{ Table 2: Oral examination findings among cases $(\boldsymbol{n}=\mathbf{8 4})$} \\
\hline Oral pathology & Prevalence $(\mathbf{\%})$ \\
\hline Submucosal fibrosis & $34(40.5)$ \\
Inadequate mouth opening & $30(35.7)$ \\
Cheek bites & $24(28.6)$ \\
Leukoplakia & $20(23.8)$ \\
Red/white patch & $16(19.0)$ \\
Lichen planus & $14(16.7)$ \\
Tooth mobility & $12(14.3)$ \\
Ulcer & $8(9.5)$ \\
Failure to heal & $8(9.5)$ \\
Fixation & $4(4.8)$ \\
Growth & $2(2.4)$ \\
Pain/paresthesia & $2(2.4)$ \\
\hline
\end{tabular}

*Items are not mutually exclusive likelihood of oral cancer or premalignant lesion. Other risk factors, namely, high salt intake $(-0.9,0.78)$, alcohol intake $(-0.7,0.36)$, poor oral hygiene $(1.4,0.17)$, and air pollution $(1.6,0.015)$ were not associated with oral cancer pathogenesis [Table 3].

\section{Discussion}

The present study suggests a high burden of oral cancer risk factors in the study population. These findings are highly significant considering majority $(82.9 \%)$ of the study participants were rural residents with poor accessibility to health care. Poor socioeconomic status of the study group is evident from low average monthly income (Rs. 1430, USD 20) and education years (3.2) as observed in past. ${ }^{[9]}$

Upon analysis, it was found that cases had a high burden of modifiable risk factors such as tobacco intake (88.1\%), dietary deficiency $(82.1 \%)$, poor oral hygiene $(35.7 \%)$, sun radiation exposure $(46.4 \%)$, pollution exposure $(22.6 \%)$, past oral lesions $(20.2 \%)$, and sepsis $(17.9 \%)$ deficient public health system for health awareness, screening and promotion aggravated by inaccessible and unregulated clinical care. Similar findings have been observed in the past by Madani et al. and Mahapatra et al. ${ }^{[10,11]}$ High salt intake (45.8\%) and alcohol intake (27.9\%) were also found in the control group, suggesting the prevailing risk of oral cancer pathogenesis in the study population.

Study project included a standard oral examination to establish the need and feasibility of setting oral cancer screening infrastructure at primary healthcare level. Such operational reforms will ensure early and quality detection of cancerous and precancerous lesions in the vulnerable populations as demonstrated in the study. In present study most common pathologies detected were submucosal fibrosis $(40.5 \%)$, inadequate mouth opening $(35.7 \%)$, cheek bites (28.6\%), and leukoplakia (23.8\%).

Multivariate logistic regression analysis suggested tobacco intake, dietary deficiency, oral sepsis, oral lesions, and sun radiation exposure had significant linkage to oral cancer pathogenesis in an independent manner. Other risk factors such as poor oral hygiene and air pollution were more in cases but were not statistically significant. Contrary to findings of a past Swedish study, the alcohol and high salt intake were more common among our study controls. However the group difference was statistically insignificant and readers discretion is advised to avoid misinterpretation. ${ }^{[12]}$

\section{Limitations of the study}

Matching of the controls was not done with the cases. Despite efforts, the recall bias (misinformation etc.) remains a major limitation in a case-control study. Complete treatment follow-up and medical assistance to the cases could not be provided due to resource constraints; however, all the due efforts were made. 


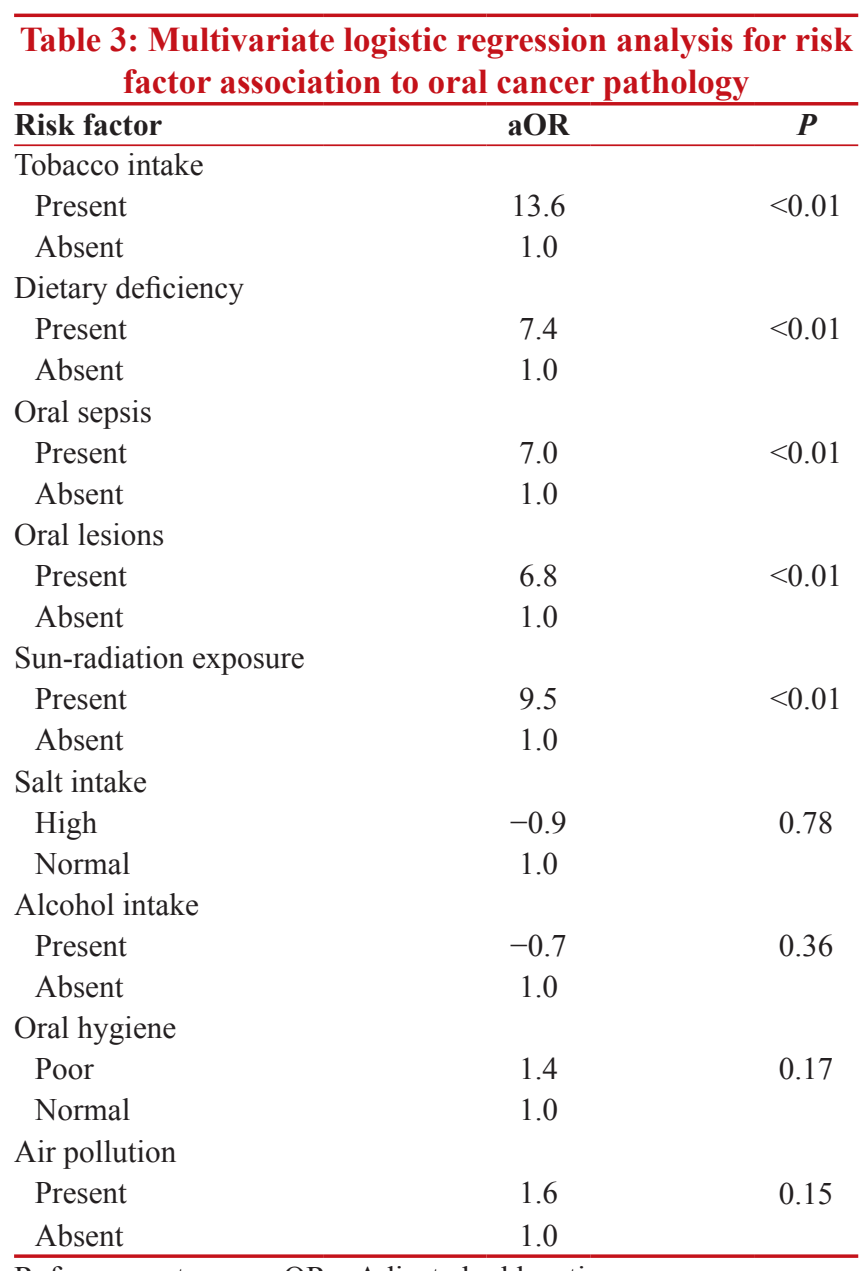

Reference category. aOR - Adjusted odds ratio

\section{Conclusion}

The present study highlights the major risk factors of oral cancer pathogenesis in studied rural population. High prevalence of conventional risk factors such as tobacco intake, solar radiation exposure, poor oral hygiene and dietary micronutrient deficiency, and disease burden indicates a huge gap in public health. The opportunistic oral screening led to successful detection and confirmation of a variety of cancerous and precancerous lesions. The study establishes the high risk of oral cancer pathogenesis due to exposure to certain risk factors such as tobacco intake, dietary micronutrient deficiency, oral sepsis and lesions, and high exposure to solar radiation.

In the rural settings where it is difficult to ensure primordial and primary disease prevention, early screening can be considered as an appropriate public health strategy. Opportunistic screening not only suffices secondary prevention but also can be conveniently used for health promotion activities. ${ }^{[13]}$ The present study reveals that it is a necessary and a practical approach to train primary care physicians in oral screening, especially in vulnerable populations. Opportunistic screening is less systematic and very cost-effective than population screening. The authors recommend all the stakeholders to unite and plan operational researches across the country for the further development of social models, strategies, and practices in this regard. ${ }^{[14]}$

\section{Acknowledgments}

The authors acknowledge the help received from the scholars whose articles are cited and included in references of this manuscript.

\section{Financial support and sponsorship}

Nil.

\section{Conflicts of interest}

There are no conflicts of interest.

\section{References}

1. Nair DR, Pruthy R, Pawar U, Chaturvedi P. Oral cancer: Premalignant conditions and screening - An update. J Cancer Res Ther 2012;8 Suppl 1:S57-66.

2. Blot WJ. Alcohol and cancer. Cancer Res 1992;52:2119s-23s.

3. British Dental Association. Opportunistic Oral Cancer Screening. BDA Occasional Paper; April, 2000.

4. Babu S, Bhat RV, Kumar PU, Sesikaran B, Rao KV, Aruna P, et al. A comparative clinico-pathological study of oral submucous fibrosis in habitual chewers of pan masala and betel quid. J Toxicol Clin Toxicol 1996;34:317-22.

5. International Institute for Population Sciences. India National Family Health Survey (NFHS-4), 2015-16. International Institute for Population Sciences; 2017.

6. Johnson NW, Warnakulasuriya KA, Partridge M, Langdon JD. Oral cancer: A serious and growing problem. Ann R Coll Surg Engl 1995;77:321-2.

7. Blot WJ, McLaughlin JK, Winn DM, Austin DF, Greenberg RS, Preston-Martin S, et al. Smoking and drinking in relation to oral and pharyngeal cancer. Cancer Res 1988;48:3282-7.

8. National Programme for Prevention and Control of Cancer, Diabetes, Cardiovascular Diseases and Stroke (NPCDCS). Available from: http:/www.health.bih.nic.in/Docs/Guidelines/ Guidelines-NPCDCS.pdf. [Last accessed on 2015 Dec 19].

9. Singh MP, Misra S, Rathanaswamy SP, Gupta S, Tewari BN, Bhatt ML, et al. Clinical profile and epidemiological factors of oral cancer patients from North India. Natl J Maxillofac Surg 2015;6:21-4

10. Madani AH, Dikshit M, Bhaduri D. Risk for oral cancer associated to smoking, smokeless and oral dip products. Indian J Public Health 2012;56:57-60.

11. Mahapatra S, Kamath R, Shetty BK, Binu VS. Risk of oral cancer associated with gutka and other tobacco products: A hospital-based case-control study. J Cancer Res Ther 2015;11:199-203.

12. Schildt EB, Eriksson M, Hardell L, Magnuson A. Oral snuff, smoking habits and alcohol consumption in relation to oral cancer in a Swedish case-control study. Int J Cancer 1998;77:341-6.

13. Villa A, Kreimer AR, Pasi M, Polimeni A, Cicciù D, Strohmenger L, et al. Oral cancer knowledge: A survey administered to patients in dental departments at large Italian hospitals. J Cancer Educ 2011;26:505-9.

14. Nunn H, Lalli A, Fortune F, Croucher R. Oral cancer screening in the Bangladeshi community of Tower Hamlets: A social model. Br J Cancer 2009;101 Suppl 2:S68-72. 